\title{
Non-periodic pseudo-random numbers used in Monte Carlo calculations
}

\author{
Gaston E. Barberis \\ (Dated: February 5, 2020)
}

\begin{abstract}
The generation of pseudo-random numbers is one of the interesting problems in Monte Carlo simulations, mostly because the common computer generators produce periodic numbers. We used simple pseudo-random numbers generated with the simplest chaotic system, the logistic map, with excellent results. The numbers generated in this way are non-periodic, which we demonstrated for $10^{13}$ numbers, and they are obtained in a deterministic way, which allows to repeat systematically any calculation. The Monte Carlo calculations are the ideal field to apply these numbers, and we did it for simple and more elaborated cases. Chemistry and Information Technology use this kind of simulations, and the application of this numbers to Quantum Monte Carlo and Cryptography is immediate. I present here the techniques to calculate, analyze and use these pseudo-random numbers, show that they lack periodicity up to $10^{13}$ numbers and that they are not correlated.
\end{abstract}

PACS numbers:

\section{INTRODUCTION}

Monte Carlo calculation is a very efficient method to simulate complex physical systems. It is based in the generation of random numbers, and the main applications of this method in physics are statistical and quantum systems. Generally, instead of true random numbers, the researchers use pseudo-random numbers, generated by computers. This has an important advantage over the use of real random numbers: every calculation can be repeated several times, if necessary, and the pseudo-random numbers generated repeatedly, in order to check previous results. The main problem with those numbers is the periodicity that appears near one billion generated numbers, together with undesirable correlations, linear and no linear, that appear in the sets. Here we present the use of the simplest equation where chaos occurs, the logistic map.[1] Using it, it is possible to generate random numbers in the $(0,1)$ interval, with a particular distribution, and using the Ulam-Neumann transformation [2-5] they transform in a set of uniform deviates in that interval. This paper is dedicated especially to the study of the pseudo-random numbers generated with the logistic map, their periodicity and correlation. The pseudo-random numbers generated with it are not periodic, allowing us to create sequences as big as necessary to simulate statistical systems. The random generated numbers, as described above, allow us a wide field of applications. The obvious among them is also the simplest magnetic system simulation, the 2D Ising ferromagnet. As this system was exactly solved by Onsager, it allows to compare the exact results, the critical exponents and properties with the simulations, so we use it as an example of application. Other, we selected the chaotic cryptography as an exercise, using a simplified ASCII symbols set. We applied the numbers to solve condensed matter systems, as colossal magneto-resistance materials, and the results of those calculations will be published elsewhere.

\section{THE PSEUDO-RANDOM NUMBERS}

The study of the logistic map where every successive number is obtained from the first with the formula

$$
x_{n+1}=r x_{n}\left(1-x_{n}\right)
$$

constitutes a subject by itself, and the fact that changing the value of $r$ transforms a very simple result in chaos is impressive to everyone who sees this for the first time. Plotting the result for $r=2.8,3.3,3.5$ etc., and finally arriving to the infinite value for the periodicity, about $r=3.57$ provides information about the evolution of the map. When $r=4.0$ the map generates random numbers distributed in the interval $[0,1]$ as shown in Fig. 1 .

The particular distribution can be easily transformed to uniform deviates using the Ulam-Neumann transformation:

$$
y_{n}=\frac{2}{\pi} \arcsin \sqrt{x_{n}}
$$

Our numbers were generated as follows: A Fortran program with the code to calculate the formulas above was written, and calculated with a seed $x_{n}$ for $n=0$. We called this number the seed of the sequence. We discarded the first 300 numbers in every calculation, to avoid any dependence of it with the initial condition.

The necessary step that follows is to demonstrate that the numbers are non-periodic, at least within the limits that we are going to use, and beyond. We check the periodicity with a small program in a PC computer, looking 


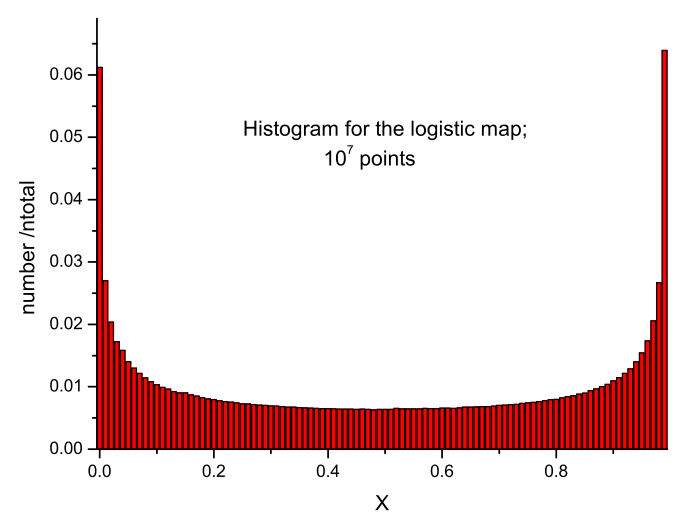

FIG. 1: Histogram showing the distribution of random numbers in the [0,1] interval, within 100 divisions.

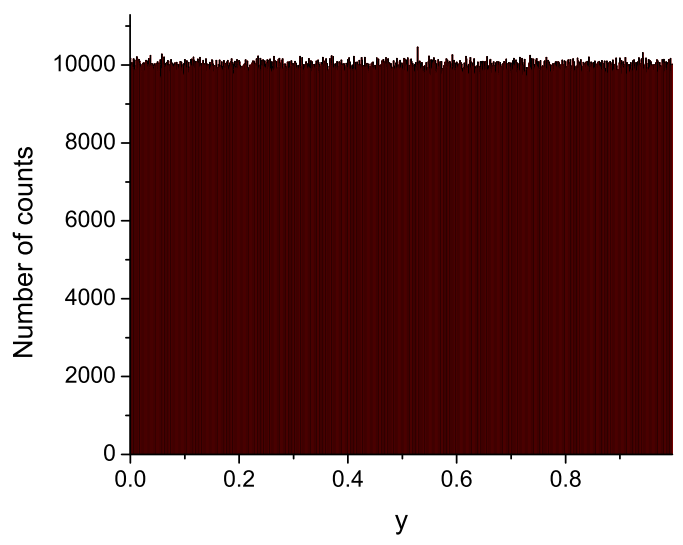

FIG. 2: Result of the Ulam-Neumann transformation as a histogram with 1000 divisions, to show the noise for $10^{7}$ numbers

for any repeated number as the first step, and we proved the numbers are non-periodic up to $10^{11}$ easily, running the program about 36 hours. As we wanted to check the periodicity beyond this limit, we used several parallel and faster computers to check it to $10^{13}$ numbers. Even limited to rational numbers, as every digital computer is, we did not observe any period in the numbers.

The Monte Carlo and other applications require sometimes various independent sets of random numbers for their development, as in the study of $2 \mathrm{D}$ or $3 \mathrm{D}$ Ising models. The question is, if our method allow the generation of independent sequences. We tried this possibility using different seeds, and we observed that every sequence obtained, as that is independent of the others, checking this result as detailed below.

Linear correlation was very easily checked. Even with a small quantity of numbers as $10^{7}$, the correlation factor is less than $10^{-6}$. Fig 3 shows a graph for a small number of data and two independent sets. Fig 4 shows the noncorrelation of the same two sets between them. Non linear correlation was also demonstrated using the Hessian matrix. The mean, variance, skewness and kurtosis of the distributions were calculated for several seeds, and the comparison through Student tests and other test demonstrated that the distributions are equal from the point of view of the parameters, and different as they are not correlated between them. All these calculations were extended for a great quantity of numbers, of the order of $10^{11}$, to make conclusive the results. It is very easy to obtain distributions of pseudo-random numbers which are exponential or normal deviates, beginning with our random deviates and transforming them [7]. Those distributions own the same properties of non-periodicity as the original one. 


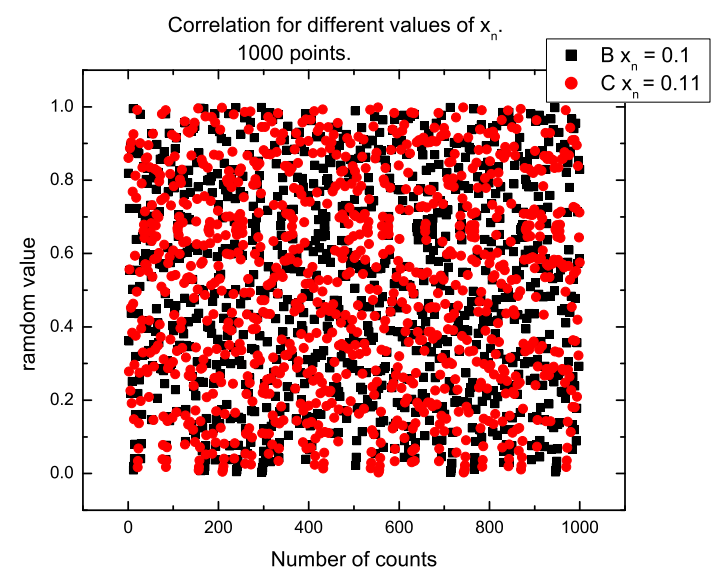

FIG. 3: Graphic to show the non-correlation between numbers of a set. The plot shows a limited number of points for clarity $\left(10^{7}\right.$ points).

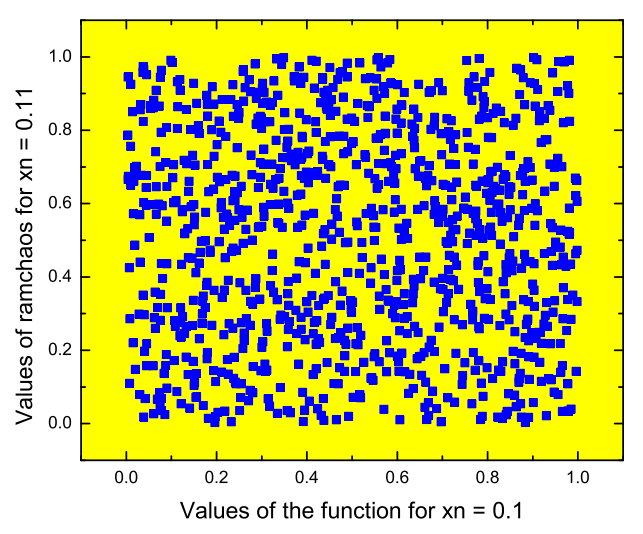

FIG. 4: The same as in Fig 3, but relating two different sets. The number of points is limited for clarity.

\section{APPLICATIONS}

The Ising model has several advantages as a check up for the pseudo-random generator. One of them is the possibility to calculate it for one, two and three dimensions using the same desk computer that we were using above. The second, is that the 2D model was solved exactly, and it is possible to use it to compare simulations with exact results, including critical parameters, and delicate properties of the system. Last, but no least, the model is not a quantum but a classic system when the magnetic field is applied parallel to the spin orientation direction, and so, it allows a statistical rather than quantum calculation. With the field perpendicular to the spin orientation, it becomes maybe the simplest quantum system to be studied. Three models, 1D, 2D and 3D [6] were calculated, and the meaning of phase transition was understood. The paradigmatic $2 \mathrm{D}$ Ising model was calculated for a $150 \times 150$ lattice, using the simplest method, the single spin-flip and the periodic boundary conditions using importance sampling, that is, the Metropolis routine[8]. We began studying a $5 \times 5,10 \times 10$ and $100 \times 100$ lattices, to study the size effect on the calculation, and we found that the $150 \times 150$ almost reproduce the exact results of Onsager. The $5 \times 5$ and the $10 \times 10$ lattices were calculated exactly, using classical statistical method, in order to compare with the simulations, and those were simulated using congruential generators, too. When the number of sites in the lattice increase, this last comparison was impossible, for the generators overpass the limits of their periodicity. The first calculation was done within a limited number of iterations for every temperature, $10^{4}$ iterations, and was afterward repeated with $10^{5}$ iterations, which was enough to obtain the magnetization curve shown in fig. 5 and the fluctuations plotted in 


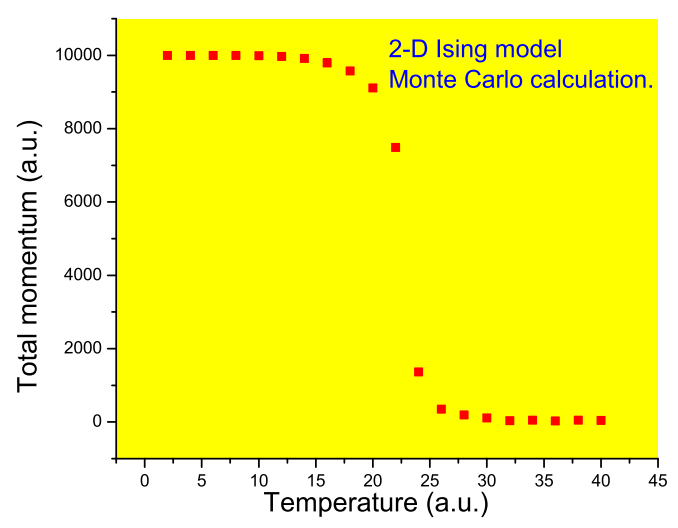

FIG. 5: (Color online)Magnetization as function of reduced temperature for the 2D Ising model. The points are both representation of the Onsager theory and the simulation, as the size is small than the error.

fig. 6. We used a single routine for all the sets, but obtained different sets just changing the seed slightly ( from .1 to .11, say). These curves are but an example of what can be done with out sets of numbers, and we do intent to demonstrate graphically the efficiency of them. All the calculations shown here were made using a PC computer, and Fortran 77 code, and for the 2D Ising model, the time necessary to complete the calculation was less than a day. Most of the - let call them "serious" calculations could require parallel processors, which make faster the calculation, but it is always possible to do modeling - calculations with a small number of iterations - with the table computer, which makes the tedious preparation of the models efficient and comfortable for the researcher.

As this paper is intended especially to present and analyze the chaotic sequences generated, we necessarily omit applications on systems as colossal magnetoresistance compounds, where we simulated the properties of them using Quantum Monte Carlo and will be published elsewhere, but we made several other calculations as tests for the numbers. The "hit or miss" method of integration was applied to obtain the number $\pi$ from the integral of a quarter of a circle, and other integration methods were repeated, both using our numbers and common congruential pseudo-random numbers [6]. In these cases, we compared the velocity for the calculations, which is less for the simple congruential, but faster when the congruential programs are more sophisticated, as the routine Ran4 in ref. [6]. Following an idea in ref. [1], we developed a simple and efficient chaotic encripter and decripter. For this purpose, we used a simplyfied version of the ASCII set, adding and substracting the numbers generated by the chaotic generator. The code worked perfectly, and the only information necessary to decript the messages is the seed. In this sense, we found that the sets generated with very small differences in the sets are not correlated, and so, a 5 cipher number could be an excellent seed for this purpose.

Many other calculations were developed to check the pseudo-random numbers. Just to mention it, we calculated the Ising model using non-periodic and screw periodic boundary condition for the clusters, identifying size effects in the calculations; we calculated the same model using cluster flipping methods, and calculated other systems, as th Heisenberg ferromagnet, using Monte Carlo methods. The results of those calculations, whenever they conduce to new results, will be publish elsewhere.

\section{CONCLUSIONS}

We conclude that we could develop computer programs using the chaotic properties of the logistic map, for $\mathrm{r}=4$, where the successive results are chaotic, deterministic and non-periodic. We studied the sets so obtained, comparing them with other sets of pseudo-random numbers obtained classically, and the conclusion is that the chaotic sets accomplish the benefits obtained with the non-chaotic sets, that is, velocity and determinism, and allow to use sets as big as necessary, because of their non-periodicity. Most of the preparation and tests were made in a PC computer, 


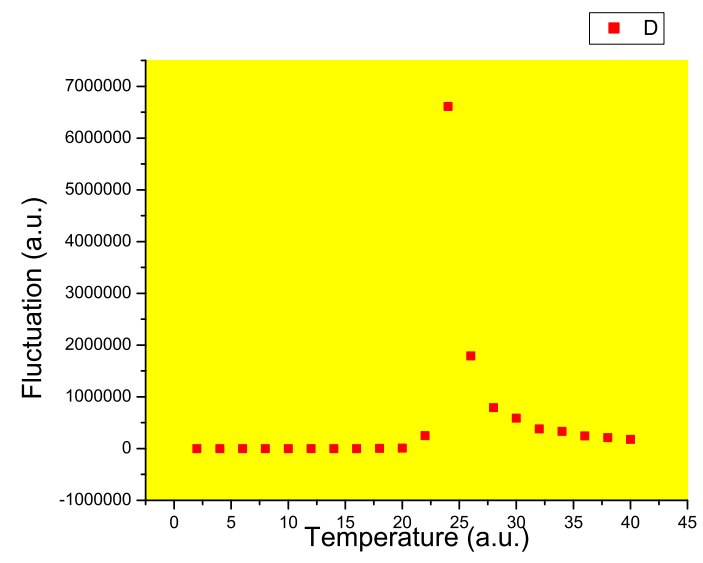

FIG. 6: (color online)Fluctuations in the 2D Ising model. As in the previous figure, the simulation and the model coincide.

which shows its practicity, even when calculations with large number of simulations require faster computers.

1 S.H. Strogatz, Nonlinear dynamics and chaos, Perseus (1994)

2 F. James, Chaos, Solitons and Fractals 6, 221 (1995)

3 J.A.Gonzalez, L.I, Reyes and L.E. Guerrero, Chaos, 11, 1 (2001)

4 D.P. Landau and K. Binder,. MC Simulation in Stat. Phys. Cambridge, (2000)

5 J.J. Collins et al., Computer in Physics, 6,630 (1992)

6 A.M. Ferrenberg and D.P. Landau, Phys. Rev. B, 44, 5081 (1991).

7 W.H. Press, S.A. Teukoisky, W.T. Vetterling and B.B. Flannery, Numerical recipes in C, U. of Cambridge Press (1995).

8 N. Metropolis, A.W. Rosenbluth, A.M. Teller and E. Teller, J. Chem. Phys. 21, 1087 (1953). 